\title{
Spectroscopic studies on the interaction of hypocrellin A with myoglobin
}

\author{
J.H. Zhou ${ }^{\text {a }}$ X.H. Wu ${ }^{\text {a }}$, C. Yang ${ }^{\text {c }}$, X.T. Gu ${ }^{\text {a }}$, L. Zhou ${ }^{\text {b }}$, K.X. Song ${ }^{\text {a }}$, \\ Y.Y. Feng ${ }^{\text {a,* }}$ and J. Shen ${ }^{\text {a,* }}$ \\ ${ }^{a}$ Analysis \& Testing Center, Jiangsu Engineering Research Center for Bio-medical Function Materials, \\ Nanjing Normal University, Nanjing, 210097, P.R. China \\ ${ }^{\mathrm{b}}$ School of Life Science, Nanjing Normal University, Nanjing, 210097, P.R. China \\ ${ }^{\mathrm{c}}$ Department for intensive instruction, Nanjing Normal University, Nanjing, 210097, P.R. China
}

\begin{abstract}
Experimental results of UV-visible absorption spectroscopy and fluorescence spectroscopy indicate that hypocrellin A, which has been studied in photodynamic therapy, can interact with the surface of myoglobin through hydrophobic forces, and form a complex. Based on the Stern-Volmer equation, the quenching constants of the process can be calculated to be $4.81 \times 10^{12} \mathrm{~L} \mathrm{~mol}^{-1} \mathrm{~s}^{-1}\left(t=25^{\circ} \mathrm{C}\right)$ and $4.54 \times 10^{12} \mathrm{~L} \mathrm{~mol}^{-1} \mathrm{~s}^{-1}\left(t=42^{\circ} \mathrm{C}\right)$ respectively, and the binding constant is $5.53 \times 10^{4} \mathrm{M}^{-1}\left(t=25^{\circ} \mathrm{C}\right)$, while the binding sites is $0.94\left(t=25^{\circ} \mathrm{C}\right)$. In addition, Electron paramagnetic resonance and fluorescence spectroscopic analysis suggests that that the quenching mechanism of the interaction process occurs through the electron transfer between hypocrellin A and myoglobin.

Keywords: Hypocrellin A, myoglobin, UV-visible absorption spectroscopy, fluorescence spectroscopy, electron paramagnetic resonance spectroscopy
\end{abstract}

\section{Introduction}

Hypocrellin A (4,9-dihydroxyperylene-3-10-quinone, HA), one kind of natural pigment, which can be extracted from Hypocrella bambuase, a parasitic fungus of Siramudinaria, has been used as a phototherapeutic agent to cure various skin diseases. It is also an orally taken folk medicine for several centuries in China [1,2]. Recently, this natural perylenequinonoid compound was found to possesses the antitumoral and antiviral activities against several types of viruses, including the human immunodeficiency virus [3]. Comparing with the presently used hematoporphyrin derivatives (HPD), it possesses several advantages, i.e. ready preparation and easy purification relative to HPD, small aggregation tendency, strong red light absorptivity and high quantum yields of singlet oxygen [4,5]. According to the photodynamic therapeutic theory, in the procedure of cure, HA or its analogues will be injected intravenously into the patients, then an incubation period for some hours is needed before the treatment with light. During this interval, HA will be bound by various proteins, and be transfer to the tumor. Thereby, for knowing the details of the binding characteristic of HA with these proteins in the photodynamic therapeutic procedure at molecular level, the interaction of HA with protein need to be further studied.

\footnotetext{
${ }^{*}$ Corresponding author: Yuying Feng, Professor, Analysis \& Testing Center, Jiangsu Engineering Research Center for Biomedical Function Materials, Nanjing Normal University, 122 Ninghai Road, Nanjing, Jiangsu, 210097, P.R. China. Fax: +86 25 83598359; E-mail: zhoujiahong@njnu.edu.cn
} 
Myoglobin is a protein of musculature, and its function is mainly to transfer oxygen, on the other hand, the photosensitizer need oxygen in the photodynamic therapeutic procedure, so myoglobin was chosen as investigative target in this paper to systematically study the interactive mechanism of HA with myoglobin under physiological conditions.

\section{Experimental}

\subsection{Reagents}

Myoglobin produced by American Sigma Chemical Co. was dissolved in the phosphate buffer solution $(0.04 \mathrm{~mol} / \mathrm{L}, \mathrm{pH} 7.4,0.10 \mathrm{~mol} / \mathrm{L} \mathrm{NaCl})$. The concentration of myoglobin was $5.7 \times 10^{-6} \mathrm{~mol} / \mathrm{L}$. HA was obtained according to the procedure described in literature [7], and the purity was confirmed as $>97 \%$ by HPLC. The $1 \mathrm{mmol} / \mathrm{L}$ HA aqueous solution was prepared by adding small amounts of concentrated DMSO solution to double distilled water, and the solutions were protected from exposure to light.

\subsection{Apparatus}

UV-visible absorption spectroscopy was achieved by Lambda 17 UV/Vis spectrophotometer (PerkinElmer, USA), and absorption spectrum from 250 to $600 \mathrm{~nm}$ was recorded. All fluorescence measurements were carried out on a LS 50 B spectrofluorimeter (Perkin-Elmer, USA) with excitation at $280 \mathrm{~nm}$. Unless otherwise stated, all experiments were carried out at room temperature $25^{\circ} \mathrm{C}$.

\subsection{Electron paramagnetic resonance (EPR) measurements}

EPR spectra were obtained using a Bruker ESP-300E spectrometer operating at room temperature, and the operating conditions were as follows: microwave bridge: X-band with $100 \mathrm{~Hz}$ field modulation; sweep width: $100 \mathrm{G}$; modulation amplitude: $1.0 \mathrm{G}$; modulation frequency, $100 \mathrm{kHz}$; receiver gain: $1 \times 10^{5}$; microwave power: $5 \mathrm{~mW}$. Samples were injected into the specially made quartz capillaries for EPR analyses, and purged with argon, air or oxygen for $30 \mathrm{~min}$ in the dark respectively according to the experimental requirements, and illuminated directly in the cavity of the ESR spectrometer with a $\mathrm{Nd}$ : YAG laser (355 nm, 5-6 ns of pulse width, repetition frequency: $10 \mathrm{~Hz}, 10 \mathrm{~mJ} /$ pulse energy).

\section{Results and discussion}

\subsection{UV-visible absorption spectroscopy}

In the region of $250 \mathrm{~nm}$ to $600 \mathrm{~nm}$, myoglobin has two characteristic absorption bands, whose maximum absorption peaks lie at $280 \mathrm{~nm}$ and $409 \mathrm{~nm}$ respectively (curve 1, Fig. 1). Upon addition of increasing amounts of HA to the aqueous solution containing a fixed concentration of myoglobin, notable changes in the UV-visible absorption spectrum of myoglobin were observed. The absorption intensity at $280 \mathrm{~nm}$ increased on increasing the concentration of HA, but the other absorption peak at $409 \mathrm{~nm}$ did not obviously change. According to the previous reports [8], the absorption peak at $280 \mathrm{~nm}$ is the absorption of amidic acid residues in myoglobin, and the absorption peak at $409 \mathrm{~nm}$ can be due to the absorption of hemachrome. Therefore, such pronounced phenomenon observed from the absorption spectrum of myoglobin suggested that the interaction occurred between the surface of myoglobin, and had no direct 


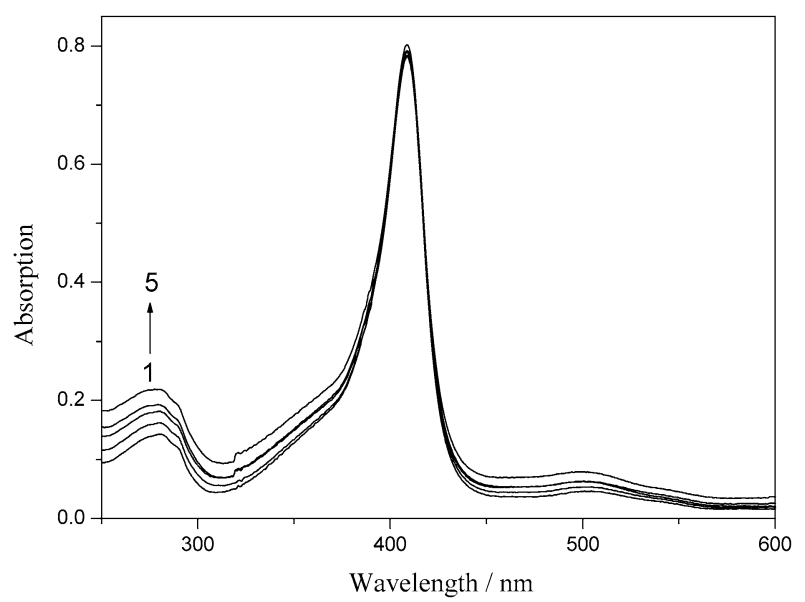

Fig. 1. UV-vis absorption spectra of $4.3 \mu \mathrm{mol} / \mathrm{L}$ Myoglobin solution with (1) 0, (2) 3.33, (3) 6.67, (4) 10.0, (5) 13.3 $\mu \mathrm{mol} / \mathrm{L}$ [HA].

effect on its interior structure. On the other hand, the controlled experiments showed that DMSO does not affect the results of above experiments under our experimental conditions, and this is consistent with the reference [9].

\subsection{Fluorescence spectroscopy}

The fluorescence quenching spectra of myoglobin with different concentrations of HA are shown in Fig. 2. It can be observed from Fig. 2 that the fluorescence peak of myoglobin is at about $330 \mathrm{~nm}$. The peak intensity is decreased and the peak position is slightly blue-shifted with increasing the concentration of HA, indicating that there is an interaction between myoglobin and HA.

There are two kinds of fluorescence quenching, i.e. the static quenching and the dynamic quenching. For dynamic quenching, it will increase the effective collision numbers, enhance the transfer of energy, and make the quenching constant of fluorescence substance augment with the increasing of temperature. If it is static quenching, along with the increasing of temperature, it will reduce the stability of the formative compound and the quenching constant. For knowing its quenching mechanism, the SternVolmer graph at various temperatures is shown Fig. 3.

Figure 3 shows that two curves have favorable linear relationships, and the slopes of the quenching curves decreased with the increasing of temperature, so it indicated that the quenching mechanism between myoglobin and HA is the static quenching.

In order to confirm the view, the procedure was assumed to be dynamic quenching. Quenching equation [10]:

$$
\frac{F_{0}}{F}=1+K_{\mathrm{s}}[Q]=1+K_{\mathrm{q}} \tau_{0}[Q]
$$

where $K_{\mathrm{q}}, K_{\mathrm{s}}, \tau_{0}$ and $Q$ are quenching rate constant of biomolecule, dynamic quenching constant, average lifetime of molecule without quencher and concentration of quencher, respectively. Because fluorescence lifetime of biopolymer is $10^{-8} \mathrm{~s}$ [11], the quenching constant can be obtained by the slope $\left(t=25^{\circ} \mathrm{C}, K_{\mathrm{q}}=4.81 \times 10^{12} \mathrm{~L} \mathrm{~mol}^{-1} \mathrm{~s}^{-1}, \gamma=0.9995 ; t=42^{\circ} \mathrm{C}, K_{\mathrm{q}}=4.54 \times 10^{12} \mathrm{~L} \mathrm{~mol}^{-1} \mathrm{~s}^{-1}\right.$, $\gamma=0.9986)$. The results indicated that the quenching constants decreased with the increasing of temperature, so the above quenching is not initiated by dynamic collision but formation of compound. In 


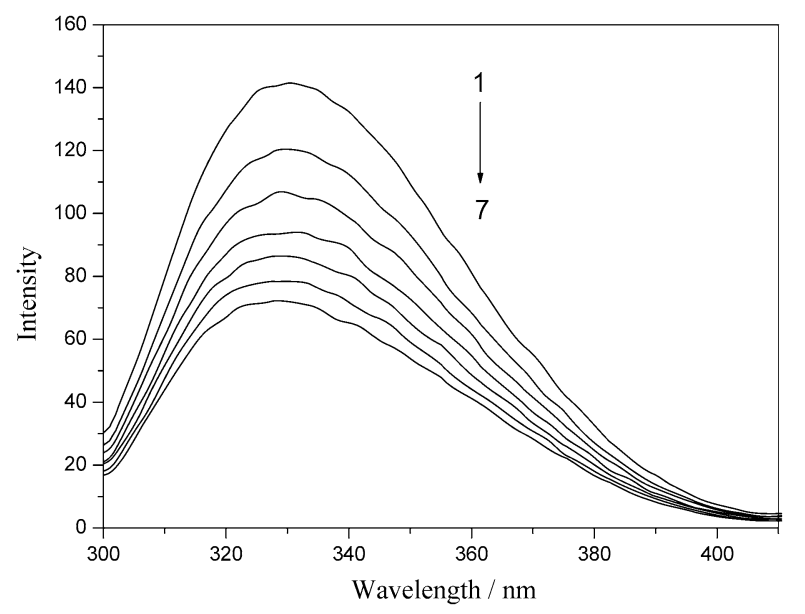

Fig. 2. Emission quenching of $5.7 \mu \mathrm{mol} / \mathrm{L}$ myoglobin solution by (1) 0 , (2) 3.33, (3) 6.67, (4) 10.0, (5) 13.3, (6) 16.7, (7) $20.0 \mu \mathrm{mol} / \mathrm{L}$ HA. $\lambda_{\mathrm{ex}}=280 \mathrm{~nm}$.

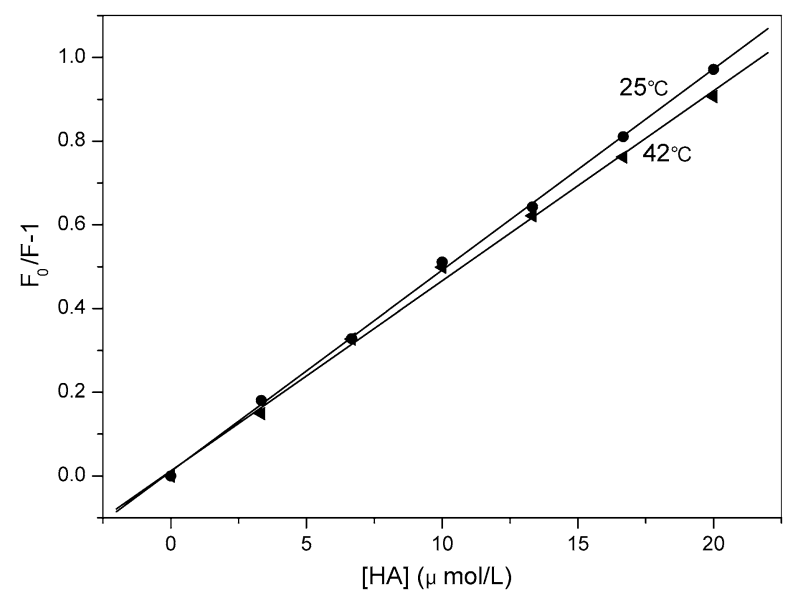

Fig. 3. The Stern-Volmer curves.

addition, the maximum scatter collision quenching constant of various quenchers with the biopolymer is $2.0 \times 10^{10} \mathrm{~L} \mathrm{~mol}^{-1} \mathrm{~s}^{-1}$ [12]. Obviously, the rate constant of protein quenching procedure initiated by HA is greater than the $K_{\mathrm{q}}$ of the scatter procedure. Thus, this also shows that the quenching is not initiated by dynamic collision but compound formation.

\subsection{Binding parameters of interaction}

The static quenching equation is [13]:

$$
\begin{aligned}
& \frac{1}{F_{0}-F}=\frac{1}{F_{0}}+\frac{K_{\mathrm{D}}}{F_{0}[Q]}, \\
& K_{\mathrm{A}}=\frac{1}{K_{\mathrm{D}}},
\end{aligned}
$$




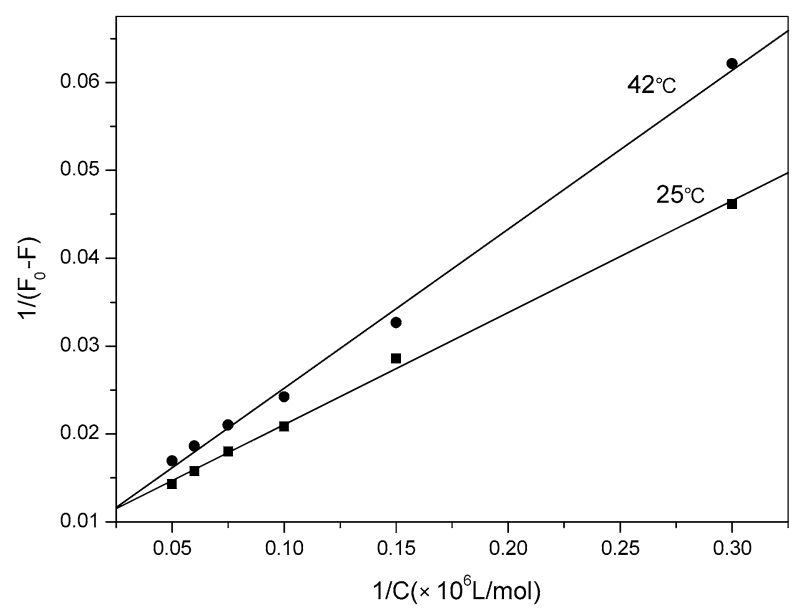

Fig. 4. The Lineweaver-Burk curves.

Table 1

Estimated values of binding constant $\left(K_{\mathrm{A}}\right)$ and possible number of binding sites $(n)$ for binding of HA and Myoglobin

\begin{tabular}{lcc}
\hline $\begin{array}{l}\text { Temperature } \\
\left({ }^{\circ} \mathrm{C}\right)\end{array}$ & $\begin{array}{c}K_{\mathrm{A}} \\
(\mathrm{mol} / \mathrm{L})^{-1}\end{array}$ & $n$ \\
\hline 25 & $5.53 \times 10^{4}(r=0.9988)$ & $0.94(r=0.9993)$ \\
42 & $4.46 \times 10^{4}(r=0.9982)$ & $1.00(r=0.9977)$ \\
\hline
\end{tabular}

where, $K_{\mathrm{A}}$ and $K_{\mathrm{D}}$ are the formation constant and the dissociation constant of the composite compound of HA and myoglobin. According to Eq. (2), $K_{\mathrm{D}}$ can be obtained from the slope of the curves in Fig. 4. Then, $K_{\mathrm{A}}$ was obtained and its data were listed in Table 1 . The results show that the binding constant between HA and myoglobin is great, so HA can be stored and removed by protein in the body.

The possible number of binding sites $(n)$ can be determined from the plot of $\lg \left[\left(F_{0}-F\right) / F\right]$ versus $\lg [\mathrm{HA}]$ according to the equation [14]:

$$
\lg \left[\frac{F_{0}-F}{F}\right]=\lg K_{0}+n \lg [\mathrm{HA}]
$$

The data obtained were also listed in Table 1.

\subsection{Binding force of interaction}

The forces acting between a drug and a biomolecule may include a hydrogen bond, van der Waals force, electrostatic force, hydrophobic interaction force, etc. If the temperature changes little, the reaction enthalpy change is regarded as a constant. According to the equation:

$$
\begin{aligned}
& \operatorname{Ln}\left(\frac{K_{1}}{K_{2}}\right)=\left(\frac{1}{T_{1}}-\frac{1}{T_{2}}\right) \frac{\Delta H}{R}, \\
& \Delta G=\Delta H-T \Delta S=-R T \ln K .
\end{aligned}
$$


In the equation, $\Delta H, \Delta G$ and $\Delta S$ are enthalpy change, free energy change, and entropy change, respectively. From the above results, these values has been calculated to be $-9.87 \mathrm{~kJ} \mathrm{~mol}^{-1},-27.06 \mathrm{~kJ} \mathrm{~mol}^{-1}$ and $57.66 \mathrm{~J} \mathrm{~mol}^{-1} \mathrm{~s}^{-1}$, and indicate that the interaction between myoglobin and HA can occur by the view of thermodynamic theory. At the same time, according to the conclusion of Ross [15], it can be deduced that the acting force between HA and myoglobin are mainly hydrophobic forces.

\subsection{Synchronous fluorescence}

Protein usually has only one emission band in the normal fluorescence spectrum, which can be due to the emission of tryptophan residues and tyrosine residues in the protein, and these two emission peaks can not be separated using normal fluorescence spectroscopy. To separate the emission of tryptophan residues and tyrosine residues, synchronous fluorescence spectroscopic technique has been introduced. According to the papers, when the wavelength interval $(\Delta \lambda)$ between the excitation and emission wavelengths $\left(\lambda_{\mathrm{ex}}\right.$ and $\left.\lambda_{\mathrm{em}}\right)$ is $20 \mathrm{~nm}$, the emission peak is due to the emission of tyrosine residues; when the value of $\Delta \lambda$ is $80 \mathrm{~nm}$, the emission peak is due to the emission of tryptophan residues [16]. For clearly knowing the quenching mechanism of myoglobin with HA, the experiments of synchronous fluorescence spectroscopy were carried out.

Figure 5 shows the relation between the fluorescence intensity of myoglobin and the concentration of $\mathrm{HA}$ at $\Delta \lambda=20 \mathrm{~nm}$ or $80 \mathrm{~nm}$. In the scan region, tyrosine residues in myoglobin have one emission peak at $309 \mathrm{~nm}$, and tryptophan residues at $350 \mathrm{~nm}$. When adding HA, all two emission intensity decreased, and the peak positions shifted to red slightly. It is well known that if the emission peaks of tyrosine residues or tryptophan residues in protein red-shift more, the exposure degree of two amino acid residues will increase, and the environment will change from hydrophobic to hydrophilic. In our experimental condition, the emission peak of tryptophan residues red-shifts about $4 \mathrm{~nm}$, and the position is at $354 \mathrm{~nm}$. It approached to the position of tryptophan in the aqueous solution alone [17], indicating that tryptophan residues in myoglobin is almost up to complete exposure, thus the interaction between myoglobin and HA has changed the transform of myoglobin markedly.
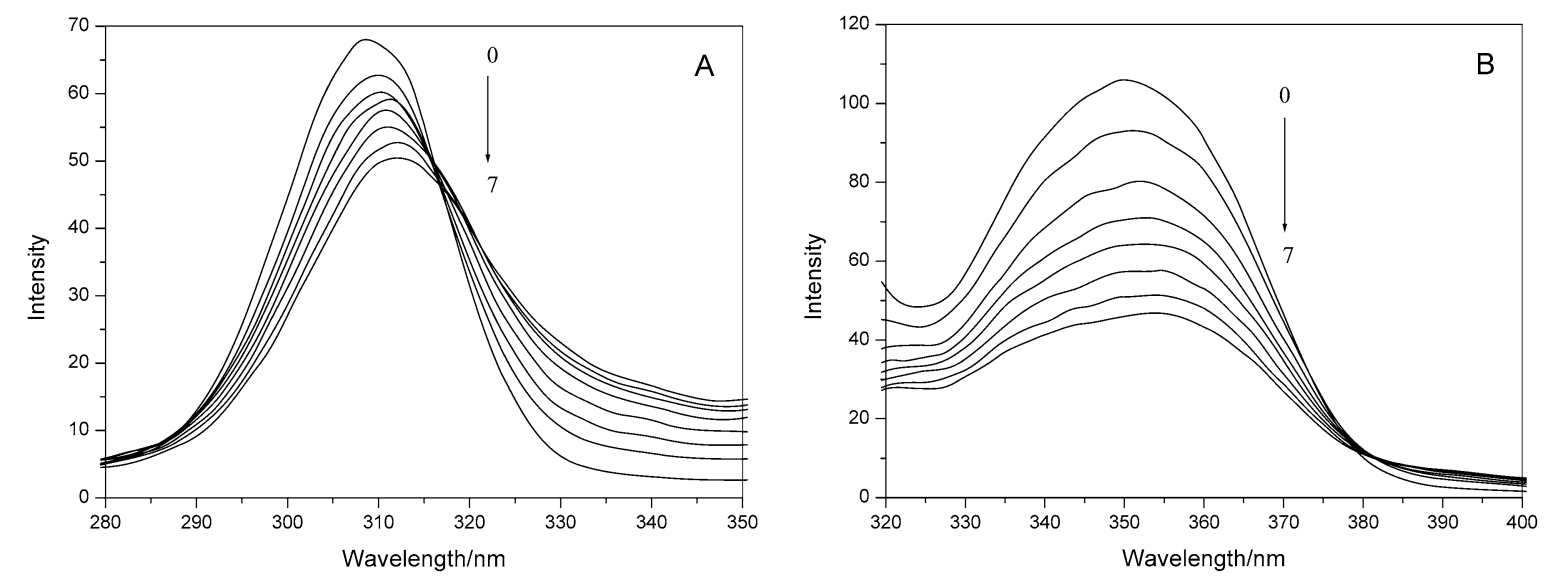

Fig. 5. Synchronous fluorescence spectra of the $5.7 \mu \mathrm{mol} / \mathrm{L}$ myoglobin solution with (0) 0, (1) 3.3, (2) 6.7, (3) 10, (4) 13.3, (5) 16.7, , (6) $20 \mu \mathrm{mol} / \mathrm{L}$ HA. $\Delta \lambda$ (A) $20 \mathrm{~nm}$, (B) $80 \mathrm{~nm}$. 


\subsection{The fluorescence quenching mechanism of Mb by HA}

If adding the solid of myoglobin into the solution of HA, and excited at $280 \mathrm{~nm}$, the fluorescence intensity of $\mathrm{HA}$ at $600 \mathrm{~nm}$ will decrease gradually with the added amount of myoglobin increasing. The results indicated that the non-radiative energy transfer is not mainly in the fluorescence quenching process of myoglobin.

For confirming the fluorescence quenching mechanism of electron transformation, EPR experiments were carried out. Irradiating the nitrogen-saturated aqueous solution of HA $(40 \mu \mathrm{M})$ for $1 \mathrm{~min}$ at $355 \mathrm{~nm}$, an EPR signal was observed (Fig. 6, spectrum a), and it is the semiquinone anion radical of HA. The formation of radical anions of HA is believed to be the result of self-electron transfer between excited and ground state HA [18].

When myoglobin was added to the deoxygenated aqueous solution of HA, the intensity of the EPR signal of the semiquinone anion radical of HA was enhanced significantly (Fig. 6, spectrum b). Bubbling air through the irradiated HA solution can make the EPR signal disappeared immediately (Fig. 6, spectrum c), on the other hand, with the absence of HA or light or nitrogen, there was not any EPR signal observed (Fig. 6, spectrum d).

The observation mentioned above can be attributed to the photoinduced electron transfer between HA and myoglobin and the formation of the semiquinone anion radical of HA. From the synchronous fluorescence results, it can be known that the electron transfer occurred between tryptophan residues or tyrosine residues and HA. The oxidation potential of tryptophan and tyrosine is $0.88 \mathrm{~V}$ and $0.93 \mathrm{~V}$, respectively [19,20], and the reduction potential of HA is $-0.37 \mathrm{~V}$ versus NHE [21]. Thus, based on their absorption edges ( $313 \mathrm{~nm}$ for tryptophan, $290 \mathrm{~nm}$ for tyrosine and $640 \mathrm{~nm}$ for HA) [22], the oxidation potentials of the excited tryptophan and tyrosine are estimated to be $-3.08 \mathrm{~V}$ and $-3.35 \mathrm{~V}$ versus NHE, respectively, and the reduction potential of the excited HA is $1.57 \mathrm{~V}$ versus NHE. So the electron transfer from excited state of tryptophan or tyrosine to ground state of HA is a thermodynamically favorable process, $(\Delta G(\operatorname{Trp})=-62.5 \mathrm{kcal} / \mathrm{mol}, \Delta G(\mathrm{Tyr})=-68.7 \mathrm{kcal} / \mathrm{mol})$ estimated by Rehm-

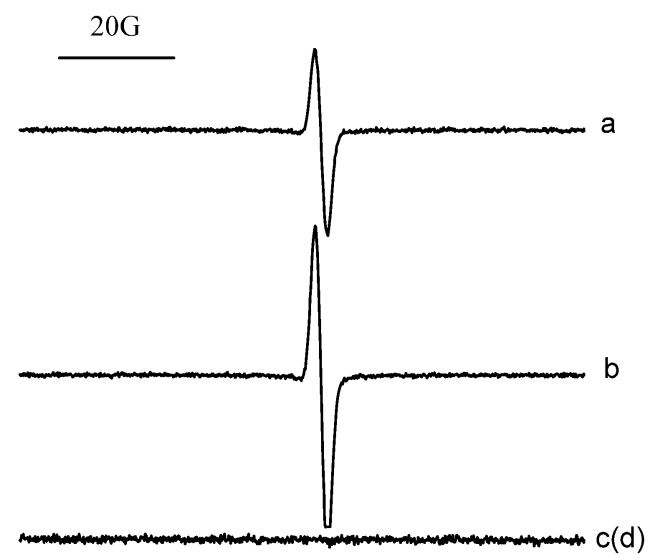

Fig. 6. Photoinduced EPR spectra of deoxygenated aqueous solution of HA (40 $\mu \mathrm{M})$ HA solution: (a) irradiation for 1 min, (b) same as (a) but in the presence of myoglobin $(40 \mu \mathrm{M})$, (c) same as (a) but oxygen was bubbled, (d) same as (a) but HA, or light was omitted. 
Weller equation where the singlet excited state of tryptophan and tyrosine are $3.96 \mathrm{eV}$ and $4.28 \mathrm{eV}$, respectively.

$$
\Delta G=E_{\mathrm{ox}}(\text { donor })-E_{\mathrm{red}}(\text { acceptor })-E_{0,0}(\text { excited state energy }) .
$$

In addition, when the electrons transfer from excited state of HA to the ground state of tryptophan or tyrosine, the singlet excited state of HA is $1.94 \mathrm{eV}, \Delta G(\operatorname{Trp})=-15.9 \mathrm{kcal} / \mathrm{mol}, \Delta G(\mathrm{Tyr})=$ $-14.8 \mathrm{kcal} / \mathrm{mol}$, so it is a thermodynamically favorable process too.

As a result, photoinduced electron transfer between HA and myoglobin gives rise to the formation of the semiquinone anion radical of HA, and leads to the fluorescence quenching of myoglobin $(\mathrm{Mb})$.

$$
\begin{aligned}
& \mathrm{HA}+\mathrm{Mb} \longrightarrow \mathrm{HA}-\mathrm{Mb} \\
& \mathrm{HA}-\mathrm{Mb} \stackrel{h v}{\longrightarrow} \mathrm{HA}^{*}-\mathrm{Mb} \\
& \mathrm{HA}^{*}-\mathrm{Mb} \stackrel{I C T}{\longrightarrow} \mathrm{HA}^{-}-\mathrm{Mb}^{+} .
\end{aligned}
$$

Or

$$
\begin{aligned}
& \mathrm{HA}-\mathrm{Mb} \stackrel{h v}{\longrightarrow} \mathrm{HA}-\mathrm{Mb}^{*} \\
& \mathrm{HA}-\mathrm{Mb}^{*} \stackrel{I C T}{\longrightarrow} \mathrm{HA}^{-}-\mathrm{Mb}^{+} .
\end{aligned}
$$

\section{Conclusions}

In summary, the experimental results indicated that there is one interaction existing between HA and myoglobin. Such interaction can quench the fluorescence of myoglobin. The binding parameters (binding constant, binding forces and possible number of binding sites) proved that this fluorescence quenching is by static mechanism. Furthermore, the results of synchronous fluorescence spectroscopy and EPR suggested that the fluorescence quenching mechanism is through the electron transfer between HA and myoglobin.

\section{Acknowledgements}

The authors are grateful for the National Natural Science Foundation of China (20603018).

\section{References}

[1] X.Y. Wan and Y.T. Chen, Hypocrellin A - a new drug for photodynamic therapy, Chin. Sci. Bull. 24 (1980), $1148-1154$.

[2] L.J. Jiang, The structures, properties, photochemical reactions and reaction mechanisms of hypocrellin (I), Chin. Sci. Bull. 21 (1990), 1608-1616.

[3] G.A. Kraus, W. Zhang, M.J. Fehr et al., Research at the interface between chemistry and virology: Development of a molecular flashlight, Chem. Rev. 96 (1996), 523-535.

[4] G.G. Miller, K. Brown, A.M. Ballangrud et al., Preclinical assessment of hypocrellin B and HB derivatives as sensitizers for photodynamic therapy of cancer: Progress update, Photochem. Photobiol. 65 (1997), 714-722. 
[5] J.B. Hudson, U. Imperial, R.P. Hauglan and Z.J. Diwu, Antiviral activities of photoactive perylenequinones, Photochem. Photobiol. 65 (1997), 352-354.

[6] F. Ricchelli, S. Gobbo, G. Jori, C. Salet and G. Moreno, Temperature-induced changes in fluorescence properties as a probe of porphyrin microenvironment in lipid membranes, Eur. J. Biochem. 233 (1995), 165-170.

[7] Y.Z. Hu, J.Y. An, L. Qin and L.J. Jiang, Studies of the triplet state properties of hypocrellin A by nanosecond flash photolysis, J. Photochem. Photobiol A 78 (1994), 247-251.

[8] A.T. Tu (ed.), Raman Spectroscopy in Biology: Principle and Applications, Wiley, New York, 1982, pp. 65-99.

[9] (a) J. Warren, M.R. Sacksteder, H. Jarosz et al., Potentiation of antineoplastic compounds by oral dimethyl sulfoxide in tumor-bearing rats, Ann. N.Y. Acad. Sci. 243 (1975), 194-208; (b) J.C. Garrido and R.E. Lagos, Dimethyl sulfoxide therapy as toxicity-reducing agent and potentiator of cyclophosphamide in the treatment of different types of cancer, Ann. N.Y. Acad. Sci. 243 (1975), 412-420; (c) P. Lonnbro and I. Wadso, Effect of dimethyl sulphoxide and some antibiotics on cultured human T lymphoma cells as measured by microcalorimetry, J. Biochem. Bioph. Methods 22 (1991), 331-336.

[10] C.C. Guo, H.P. Li, X.B. Zhang and R.B. Tong, Synthesis of meso-5,10,15,20-Tetra[4-(N-pyrrolidinyl)phenyl] porphyrin and its interaction with bovine serum albumin, Chem. Res. Chinese U. 24 (2003), 282-287.

[11] J.R. Lakowicz and G. Weber, Quenching of fluorescence by oxygen. Probe for structural fluctuations in macromolecules, Biochemistry 12 (1973), 4161-4170.

[12] W.R. Ware, Oxygen quenching of fluorescence in solution: an experimental study of the diffusion process, J. Phys. Chem. 66 (1962), 455-458.

[13] M.M. Yang, X.L. Xi and P. Yang, Studies on interaction of new medicine of quinolone-class with HSA and BSA by using fluorescence enhancement and fluorescence quenching theory, Chem. J. Chinese U. 27 (2006), 687-691.

[14] H.R. Zhang, Q.Y. Guo et al., Study on the interaction between sparfloxacin and serum albumins by fluorescence, Spectrosc. Spect. Anal. 21 (2001), 829-832.

[15] S.B. Mauricio and L.I. Guilherme, Effect of BSA binging on photophysical and photochemical properties of triaylmethane dyes, J. Phys. Chem. 102 (1998), 4678-4688.

[16] D.P. Ross and S. Sabramanian, Thermodynamics of protein association reaction: forces contribution to stability, Biochemistry 20 (1981), 3096-3102.

[17] J. Chou, X.G. Qu, T.H. Lu and Y. Wu, Study of interaction between cytochrome C and cystine using synchronous fluorescence spectroscopy, Spectrosc. Spect. Anal. 17 (1997), 108-112.

[18] J.H. Zhou, S.Q. Xia, J.R. Chen, X.S. Wang and B.W. Zhang, The photodynamic property improvement of hypocrellin A by chelation with lanthanum ions, Chem. Commun. 12 (2003), 1372-1373.

[19] Z.J. Diwu, L.J. Jiang and M.H. Zhang, The mechanism of hypocrellin A sensitized photooxidation reaction, Acta Chimica Sinica 48 (1990), 483-489.

[20] S.Q. Xia, J.H. Zhou, J.R. Chen, X.S. Wang and B.W. Zhang, A tyrosine-modified hypocrellins B with affinity for and photodamaging ability towards calf thymus DNA, Chem. Commun. 23 (2003), 2900-2901.

[21] J.H. Zhou, K.W. Wu, S.Q. Xia, Z.Z. Qu, X.S. Wang, B. W. Zhang and Y. Cao, The behavior of the semiquinone anion radical of hypocrellin B produced by electrolysis in dimethylsulfoxide, Res. Chem. Intermed. 30 (2004), 439-449.

[22] Z.L. Zhu, S.G. Mi, P.S. Cong and X.H. Wu, The study on the effect of overlapped degree of spectra on accuracy in $\mathrm{pH}$ distribution-absorption spectra, Computers and Applied Chemistry 19 (2002), 60-64. 


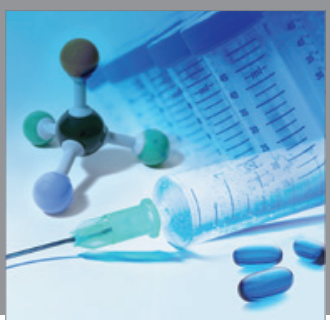

International Journal of

Medicinal Chemistry

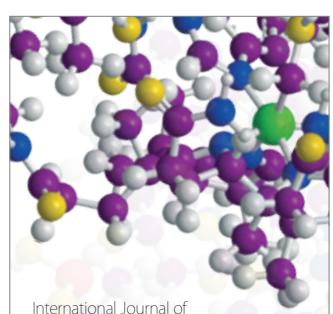

Carbohydrate Chemistry

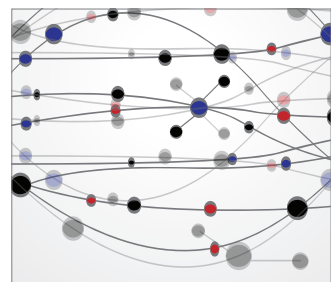

The Scientific World Journal
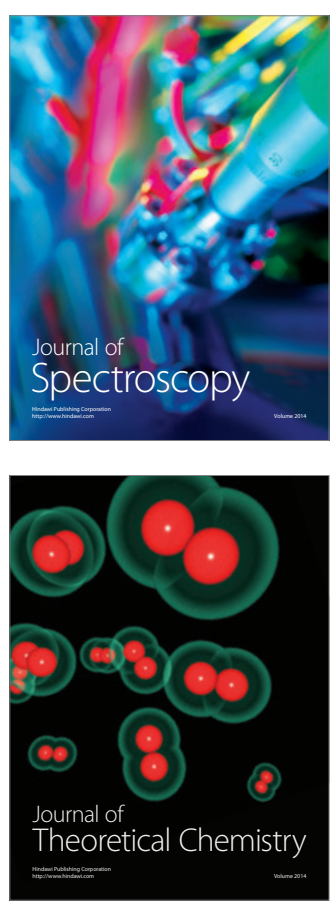
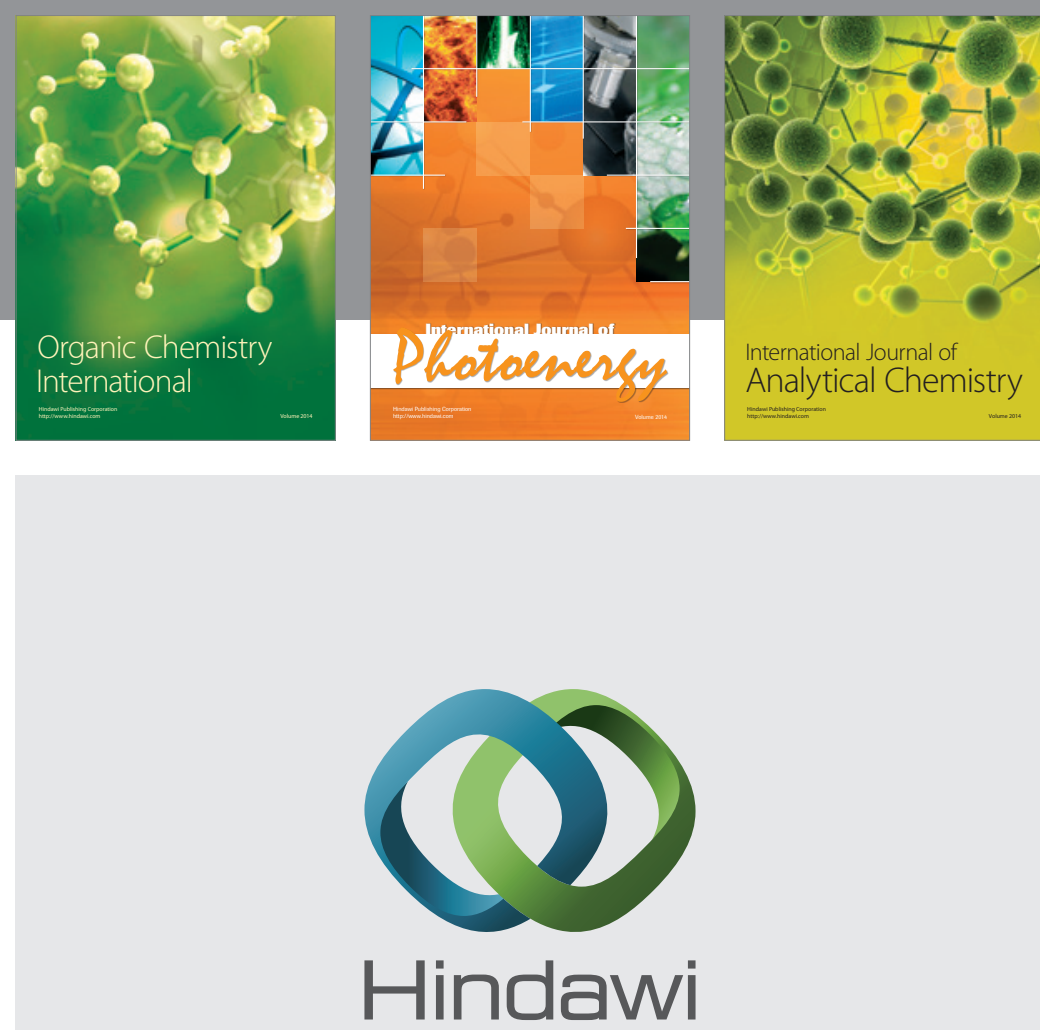

Submit your manuscripts at

http://www.hindawi.com
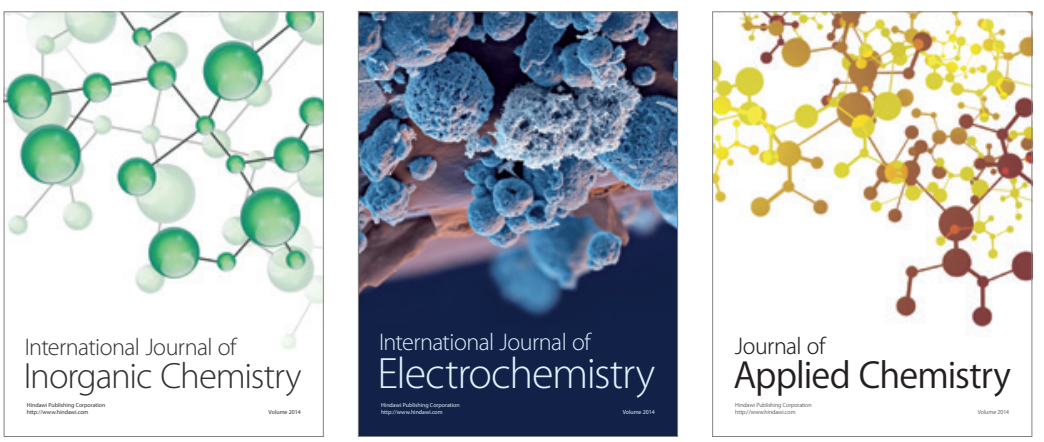

Journal of

Applied Chemistry
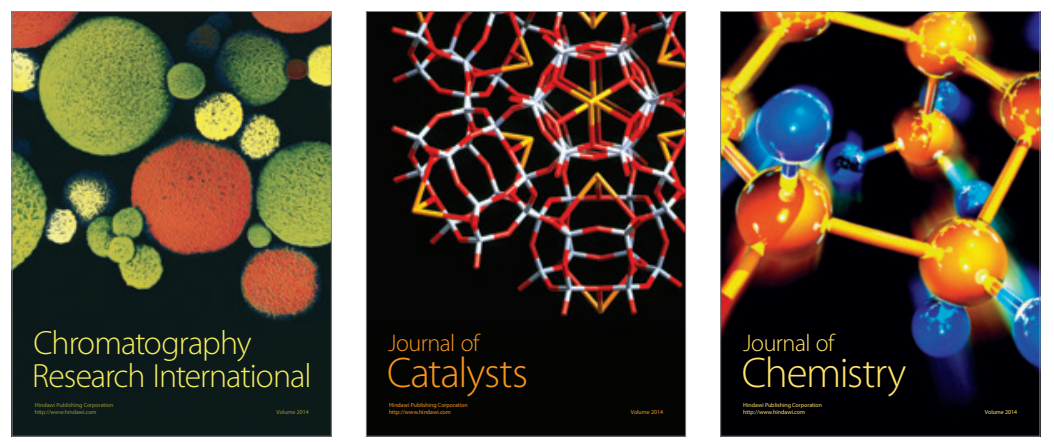
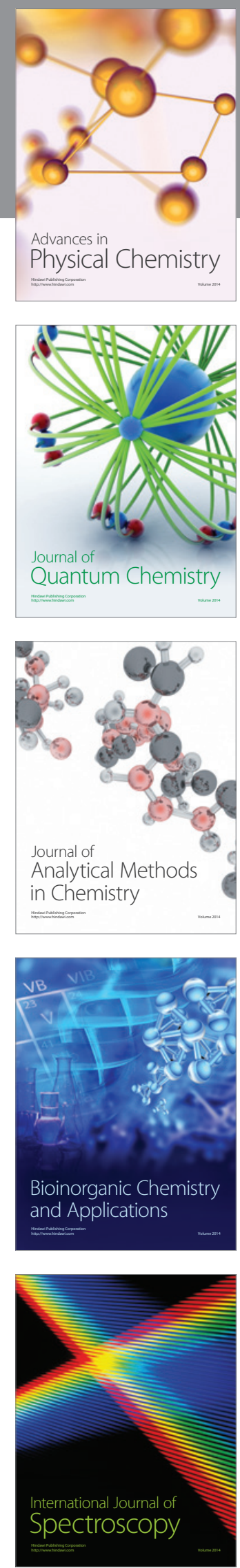